\section{ORIGINAL RESEARCH}

H.S. Seo

D.G. Na

J.-h. Kim

K.W. Kim

K.-R. Son

\title{
Correlation between CT and Diffusion-Weighted Imaging of Acute Cerebral Ischemia in a Rat Model
}

BACKGROUND AND PURPOSE: The quantitative temporal relationship between changes in CT attenuation, ADC value, and DWI signal intensity of acute ischemic tissue has not yet been determined in an animal model. This study was performed to determine the temporal relationship between CT attenuation, ADC value, and DWI signal intensity in acute cerebral ischemia.

MATERIALS AND METHODS: CT and DWI were performed at 1, 3, 5, 7, and 9 hours after left MCA occlusion in 11 rats. Mean values for CT attenuation, ADC, and DWI signal intensity were determined for the ischemic hemisphere and contralateral normal hemisphere. Temporal changes in each mean value and the relationship between CT attenuation and ADC value and DWI signal intensity were evaluated.

RESULTS: The decrease of CT attenuation and the increase of DWI signal intensity occurred gradually after MCA occlusion, while ADC value decreased rapidly at 1 hour. Although correlation was significant between time and $\mathrm{rCT}$ or rDWI $(P<.01$, respectively), no correlation between time and rADC was found $(P=.33)$. There was a significant linear correlation between $\mathrm{rCT}$ and $\mathrm{rDWI}(r=0.497, P<.01)$ but no significant correlation between $\mathrm{rCT}$ and $\mathrm{rADC}(P=.509)$ was found

CoNCLUSIONS: The temporal change in CT attenuation was different from that in ADC value with no significant linear correlation between $\mathrm{CT}$ attenuation and ADC value for acute cerebral ischemia. However, rCT and rDWI showed a modest correlation.

ABBREVIATIONS: $\mathrm{ADC}=$ apparent diffusion coefficient; $\mathrm{CBF}=$ cerebral blood flow; $\mathrm{CNR}=$ contrast-to-noise ratio; $\mathrm{dADC}=$ difference of $\mathrm{ADC}$ value; $\mathrm{dCT}=$ difference of $\mathrm{CT}$ attenuation; $\mathrm{DWI}=$ diffusion-weighted imaging; $\mathrm{HU}=$ Hounsfield units; $\mathrm{MCA}=$ middle cerebral artery; $\mathrm{ADC}=$ relative $A D C$ value; $r C T=$ relative $C T$ attenuation; $r D W I=$ relative $D W I$ signal intensity

n acute ischemic stroke, parenchymal lesions showing hypoattenuation by CT indicate severe ischemic edema, critical hypoperfusion, and irreversible ischemic injury. ${ }^{1,2}$ The water content of ischemic brain tissue is a marker of severe ischemic injury, ${ }^{3-5}$ and CT attenuation is linearly proportional to tissue water content. ${ }^{6}$ The extent of CT hypoattenuation or ischemic brain edema is associated with a poor clinical outcome and a risk of brain hemorrhage if the patient is treated with thrombolysis. ${ }^{7-9}$

DWI and ADC maps are highly sensitive for detecting acute infarctions. Decreased diffusion can be observed in ischemic brain tissue as early as 30 minutes after arterial occlusion. ${ }^{10,11}$ CT signs of parenchymal hypoattenuation develop with severe CBF decrease because early ischemic edema with uptake of water occurs when $\mathrm{CBF}$ is $<10-15 \mathrm{~mL} / \mathrm{g} / \mathrm{min}^{3}$ The $\mathrm{CBF}$ threshold for a decrease in ADC is higher than that for CT, though a decrease in ADC occurs before the CBF declines to the critical threshold for energy failure. ${ }^{12,13} \mathrm{CT}$ hypoattenua-

Received May 28, 2010; accepted after revision August 23.

From the Department of Radiology (H.S.S., D.G.N., J.-h.K., K.W.K., K.-R.S.), Seoul National University College of Medicine, Seoul, Korea; Department of Radiology (H.S.S.), Korea University Ansan Hospital, Ansan-si, Kyunggi-do, Korea; and Department of Radiology (D.G.N.), Human Medical Imaging and Intervention Center, Seoul, Korea.

This work was supported by grant 03-2006-026-0 from the Seoul National University Hospital Research Fund and, in part, by a grant from Schering $A G$.

Please address correspondence to Dong Gyu Na, MD, Department of Radiology, Human Medical Imaging and Intervention Center, 12-25, Jamwon-dong, Seocho-gu, Seoul, 137902, Korea; e-mail: nndgna@gmail.com

DOI 10.3174/ajnr.A2362 tion of acute ischemic tissue is irreversible, though permanent or transient reversal of ADC after early reperfusion has been shown in animal and human studies. ${ }^{14-17}$ However, the threshold of ADC value for predicting reversibility has not yet been determined, ${ }^{18}$ and the predictive value of ADC for determining the severity of ischemic edema is not clear. ${ }^{18,19}$

ADC values in acute ischemia have been known to decrease within the first few minutes of artery occlusion, reach the maximum decrease after 1-2 hours, and remain stable thereafter. ${ }^{20-27}$ The temporal evolution of ADC in acute ischemia has been reported in many experimental ${ }^{20-24}$ and clinical studies, ${ }^{25-27}$ whereas that of CT attenuation has been reported in few studies. ${ }^{6,27}$ The evolution of CT attenuation in acute ischemia is a continuous linear decrease. However, the quantitative temporal relationship between changes in CT attenuation, ADC value, and DWI signal intensity has not yet been established in an animal model, though the relationship between $\mathrm{CT}$ attenuation and ADC value has been investigated in some clinical studies. ${ }^{19,27}$ Therefore, we used a permanent MCA occlusion rat model to determine the temporal relationship between CT attenuation, ADC value, and DWI signal intensity in acute cerebral ischemia.

\section{Materials and Methods}

\section{MCA Occlusion Method}

This study used a total of 18 male Sprague-Dawley rats weighing $>350$ g. The intraluminal suture occlusion method proposed by Longa et $\mathrm{al}^{28}$ was used for left MCA occlusion. We excluded 4 rats that 
did not show evident territorial cerebral ischemic change on DWIs and 3 rats that expired in 5-7 hours after the procedure and did not undergo the scheduled image acquisitions within 9 hours of the procedure. Therefore, 11 rats, in which focal cerebral ischemia was successfully induced, were included in this study.

Rats were anesthetized intramuscularly with $1 \mathrm{~mL} / 100 \mathrm{~g}$ of body weight of a mixture of ketamine hydrochloride $(5 \mathrm{mg} / 100 \mathrm{~g})$ and xylazine hydrochloride $(1 \mathrm{mg} / 100 \mathrm{~g})$. The left common carotid artery was exposed through an approximately $3-\mathrm{cm}$ midline incision, performed with the rat in the supine position. The left common carotid artery and the left external carotid artery were ligated with a 6-0 silk suture and an 18-gauge Medicut cannula (Covidien, Leeds, United Kingdom) was inserted in the left common carotid artery distal to the ligation and advanced into the left internal carotid artery. A 3-0 monofilament nylon suture, on which a rounded tip had been generated by heating near a flame, was introduced into the left common carotid artery lumen through the previously inserted Medicut. The suture was advanced into the left internal carotid artery until resistance was felt, indicating that the tip had passed the MCA origin and reached the proximal anterior cerebral artery. In this way, blood flow to the MCA was blocked by the occlusion of all blood sources from the internal carotid artery and anterior cerebral artery. The neck incision was then closed.

\section{Imaging Data Acquisition}

CT and MR imaging were performed at 1, 3, 5, 7, and 9 hours after MCA occlusion. CT scanning was performed first, and MR imaging was performed immediately afterward, with no time delay. During image acquisition, rats were fixed on a hard board in a prone position under anesthesia. Coronal images of 2.5-mm thickness with no gap parallel to the skull base were obtained at CT and MR imaging. The same section location and imaging plane was obtained by adjustment, by using the common anatomic landmarks of the calvaria and the skull base on CT scout and midline sagittal MR images.

Unenhanced CT imaging was performed with an 8-channel multidetector CT scanner (LightSpeed Ultra; GE Healthcare, Milwaukee, Wisconsin) by using a sequential single-section technique. Parameters were $140 \mathrm{kV}$ (peak), $75 \mathrm{mAs}$, and an FOV of $6 \times 6 \mathrm{~cm}$. MR imaging (Signa 3T; GE Healthcare) was performed with an 8-channel head coil. DWI parameters were $b=0$ and 1000; FOV, $8 \times 8 \mathrm{~cm}$; matrix, $64 \times 128$; NEX, 8; TR/TE, 6000/79 ms. ADC maps were generated from 2-point analysis on a pixel basis, and ADC values were calculated by using the standard equation:

$$
A D C=\ln \left(S_{0} / S_{1}\right) /\left(\mathrm{b}_{1}-\mathrm{b}_{0}\right),
$$

where $\mathrm{S}_{0}$ and $\mathrm{S}_{1}$ are the 2 DWI signals at $b=0$ and $1000, b_{0}=0$, and $b_{1}=1000$.

Bolus-tracking perfusion-weighted MR images with spin-echo echo-planar imaging were obtained with TR/TE of 2000/60 ms and NEX of 1 . The decreased cerebral perfusion in the affected hemisphere was confirmed by using region-of-interest analysis of signal intensity on perfusion MR images at the first 1-hour time point after MCA occlusion. Conventional fast spin-echo T2-weighted images with an FOV of $8 \times 8 \mathrm{~cm}$, matrix of $256 \times 192$, NEX of 5, and TR/TE of $4500 / 124.3 \mathrm{~ms}$ were obtained to verify the final infarction only at the last time point after MCA occlusion.

\section{Image Analysis}

Circular regions of interest on CT and MR images were measured by a neuroradiologist (H.S.S). Regions of interest were manually posi-

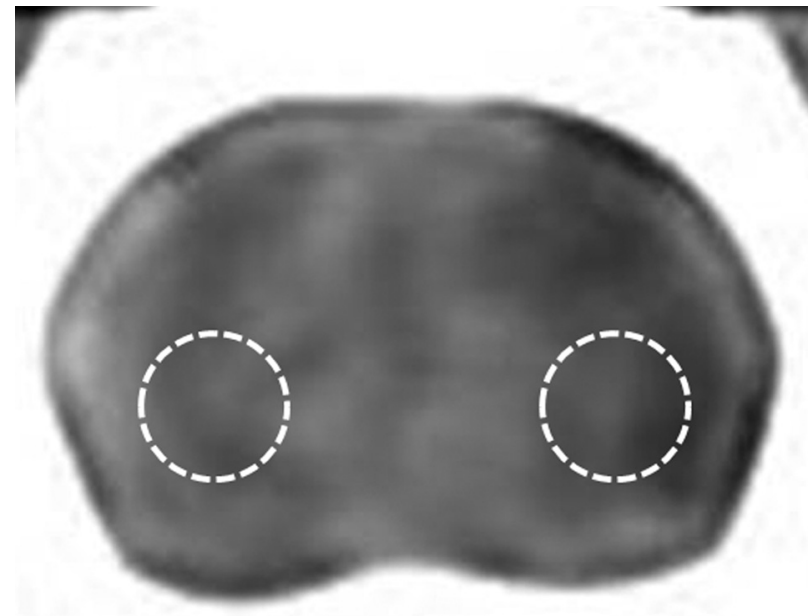

Fig 1. Measurement of the region of interest for CT attenuation in ischemic and normal brain tissue. The region of interest is positioned in the inferolateral part of the hemisphere on each CT or MR image, $5 \mathrm{~mm}$ ventral to the external auditory canal.

tioned in the inferolateral part of the left ischemic cerebral hemisphere, ${ }^{6}$ and the corresponding mirror regions of interest for each CT or MR imaging were placed at the right contralateral cerebral hemisphere $5 \mathrm{~mm}$ ventral to the external auditory canal for each case (Fig 1). Region-of-interest size was $6.6 \mathrm{~mm}^{2}$. After regions of interest were determined on a CT image, the same region of interest was reproducibly placed at the same brain location on DWI and the ADC map by carefully comparing with the same anatomic landmarks in the CT brain image. The rCT was calculated by dividing the $\mathrm{CT}$ attenuation of the ischemic cerebral hemisphere by the value for the contralateral normal hemisphere. ADC value and DWI signal intensity of the left ischemic cerebral hemisphere were measured in the same manner. rDWI and $\mathrm{rADC}$ were calculated in the same manner as for $\mathrm{rCT}$ as the ratio of the DWI signal intensity or the ADC value for the ischemic cerebral hemisphere to the value for the normal contralateral hemisphere. dCT was calculated as the difference in region-of-interest value between the left ischemic cerebral hemisphere and the normal contralateral hemisphere. CNRs for CT attenuation, $\mathrm{ADC}$ value, and DWI signal intensity were calculated as $[(A-B) / B] \times 100$, where $A$ was the CT attenuation, ADC value, or DWI signal intensity in the region of ischemic tissue and $B$ was the value for the contralateral normal tissue.

\section{Statistical Analysis}

Statistical analysis was performed with commercially available software, the Statistical Package for the Social Sciences-PC, Version 12.0; (SPSS, Chicago, Illinois). $P$ values $<.05$ were considered statistically significant. The normality of each variable was tested by using the Kolmogorov-Smirnov test. For statistical analysis of region of interest-based data, repeated-measure analyses with post hoc analysis were used to assess the longitudinal changes of mean $\mathrm{rCT}$, rDWI, and rADC values at each time point after MCA occlusion. A paired $t$ test was used to compare the CNRs among CT, DWI, and ADC maps. When the normality of the variable was not rejected, we used linear regression analysis to evaluate the correlation among $\mathrm{rCT}$, rDWI, rADC, dCT, and AADC without considering time points. 


\begin{tabular}{|c|c|c|c|c|c|}
\hline Hour & rCT & rDWI & rADC & $\mathrm{dCT} T^{\mathrm{b}}$ & $\mathrm{dADC}^{\mathrm{c}}$ \\
\hline 1 & $0.94 \pm 0.04$ & $1.40 \pm 0.07$ & $0.62 \pm 0.08$ & $2.00 \pm 1.48$ & $225.73 \pm 52.60$ \\
\hline 3 & $\begin{array}{c}0.87 \pm 0.05 \\
(P<.01)\end{array}$ & $\begin{array}{c}1.60 \pm 0.10 \\
(P<.01)\end{array}$ & $\begin{array}{c}0.55 \pm 0.06 \\
(P=.04)\end{array}$ & $\begin{array}{c}4.09 \pm 1.76 \\
(P=.01)\end{array}$ & $\begin{array}{c}268.18 \pm 43.21 \\
(P=.06)\end{array}$ \\
\hline 5 & $\begin{array}{c}0.81 \pm 0.06 \\
(P=.03)\end{array}$ & $\begin{array}{c}1.70 \pm 0.13 \\
(P<.01)\end{array}$ & $\begin{array}{c}0.56 \pm 0.04 \\
(P=.93)\end{array}$ & $\begin{array}{c}6.18 \pm 2.44 \\
(P=.04)\end{array}$ & $\begin{array}{c}262.45 \pm 28.54 \\
(P=.71)\end{array}$ \\
\hline 7 & $\begin{array}{c}0.73 \pm 0.07 \\
(P<.01)\end{array}$ & $\begin{array}{c}1.71 \pm 0.23 \\
(P=.82)\end{array}$ & $\begin{array}{c}0.58 \pm 0.06 \\
(P=.09)\end{array}$ & $\begin{array}{c}8.91 \pm 2.51 \\
(P<.01)\end{array}$ & $\begin{array}{c}250.55 \pm 42.30 \\
(P=.35)\end{array}$ \\
\hline 9 & $\begin{array}{c}0.71 \pm 0.08 \\
(P=.46)\end{array}$ & $\begin{array}{c}1.77 \pm 0.28 \\
(P=.39)\end{array}$ & $\begin{array}{c}0.57 \pm 0.08 \\
(P=.51)\end{array}$ & $\begin{array}{c}9.09 \pm 2.39 \\
(P=.84)\end{array}$ & $\begin{array}{c}257.73 \pm 73.12 \\
(P=.71)\end{array}$ \\
\hline
\end{tabular}

a Data are mean \pm SD. The figures in parentheses refer to the $P$ values compared with the value of the previous time point in post hoc analysis of repeated-measure analysis.

b dCT is in Hounsfield units.

${ }^{c} \mathrm{dADC}$ values are $\times 10^{-6} \mathrm{~mm}^{2} / \mathrm{s}$

Table 2: The mean CNRs of CT attenuation, ADC value, and DWI
signal intensity after MCA occlusion ${ }^{\mathbf{a}}$
\begin{tabular}{lrrrrrc}
\hline Hour & \multicolumn{1}{c}{ CT (\%) } & \multicolumn{1}{c}{$P^{\text {b }}$} & ADC (\%) & \multicolumn{1}{c}{$P^{\text {c }}$} & DWI (\%) & $P^{\text {d }}$ \\
\hline 1 & $6.2 \pm 4.4$ & $<.001$ & $38.5 \pm 8.4$ & .453 & $39.9 \pm 7.1$ & $<.001$ \\
3 & $12.6 \pm 4.5$ & $<.001$ & $44.8 \pm 6.1$ & $<.001$ & $59.6 \pm 9.8$ & $<.001$ \\
5 & $18.9 \pm 6.4$ & $<.001$ & $44.4 \pm 4.0$ & $<.001$ & $69.9 \pm 13.2$ & $<.001$ \\
7 & $27.1 \pm 6.5$ & $<.001$ & $41.8 \pm 6.0$ & .004 & $71.2 \pm 22.8$ & $<.001$ \\
9 & $29.0 \pm 8.0$ & .003 & $43.1 \pm 7.9$ & .002 & $76.9 \pm 28.2$ & $<.001$ \\
\hline
\end{tabular}

a Data are mean \pm SD.

${ }^{\mathrm{b}} \mathrm{CT}$ attenuation versus ADC value.

${ }^{c}$ ADC value versus DWI signal intensity.

${ }^{\mathrm{D}}$ DWI signal intensity versus CT attenuation.

\section{Results}

Table 1 gives mean region-of-interest values for rCT, rDWI, rADC, dCT, and AADC in ischemic tissue after MCA occlusion. The mean absolute region-of-interest values for CT attenuation, DWI signal intensity, and ADC value of the affected hemispheres were significantly different from those for the contralateral normal hemispheres at all time points after MCA occlusion $(P \leq .01)$. Figure 2 shows longitudinal changes in ischemic lesions on CT, DWI, and the ADC map for each time point after MCA occlusion.

\section{Relationship between CT Attenuation, ADC Value, and DWI Signal Intensity with Time after MCA Occlusion}

Figure 3 shows time courses for mean rCT, rADC, and rDWI values after the onset of cerebral ischemia. $\mathrm{rCT}$ and $\mathrm{rDWI}$ were linearly correlated to time until 9 hours after MCA occlusion with significance $(P<.01$, respectively). No significant correlations were seen between time and $\operatorname{rADC}(P=.33)$. The mean region-of-interest value for $\mathrm{rCT}$ decreased gradually but significantly, compared with each previous time point after MCA occlusion, until 7 hours $(P \leq .03$, respectively). However, no significant difference was seen in rCT between 7 and 9 hours $(P=.46)$. Mean $\mathrm{rDWI}$ values gradually increased in a significant manner until 5 hours $(P<.01$, respectively), with no significant differences in rDWI at 5,7 , and 9 hours $(P \geq .39$, respectively). The rADC rapidly and significantly decreased in the first hour and slowly up to 3 hours ( 1 versus 3 hours, $P=$ $.04)$. However, no significant differences were seen at $3,5,7$, or 9 hours ( $P \geq .09$, respectively).

dCT was also linearly correlated to time $(P \leq .01)$ and decreased gradually until 7 hours with significance $(P \leq .04$, respectively). However, no significant difference was seen in dCT between 7 and 9 hours $(P=.84)$. dADC was not linearly correlated to time $(P=.30)$, with no significantly different time interval $(P>.06$, respectively).

\section{Correlation between CT Attenuation and ADC Value or DWI Signal Intensity}

Although a significant negative linear correlation between rDWI and CT (rCT, $r=0.497, P<.01)$ and dCT $(r=0.542$, $P<.01$ ) was found (Fig 4 ), no significant linear correlation was observed between ADC (dADC or rADC) and CT (rCT or $\mathrm{dCT})(P>.50$, respectively; Fig 5). No significant correlation was also seen between ADC and CT (dADC versus $\mathrm{dCT}$ or rADC versus $\mathrm{rCT}$ ) during early periods of MCA occlusion up to 3 or 5 hours $(P>.30$, respectively).

\section{Comparison of CNRs among CT, DWI, and ADC}

At all time points, CNR for either DWI or ADC was significantly higher than that for CT $(P<.01)$ (Table 2$)$. Although the mean CNR of DWI was similar to that for ADC $(38.5 \pm 8.4$ and $39.9 \pm 7.1, P=.453)$ at 1 hour after MCA occlusion, the mean CNR for DWI was significantly higher than that for $\mathrm{ADC}$ at other time points $(P \leq .004)$.

\section{Discussion}

This study demonstrated differences between the time course for CT attenuation and ADC in acute cerebral ischemia. CT attenuation gradually decreased, while ADC rapidly decreased in the first hour after MCA occlusion. A modest correlation was observed between CT attenuation and DWI signal intensity, but no significant correlation was found between CT attenuation and $\mathrm{ADC}$ value.

A previous clinical study of acute ischemic stroke by Kucinski et $\mathrm{al}^{27}$ demonstrated a modest correlation between $\mathrm{CT}$ attenuation and ADC value in acute ischemic tissue. They suggested different time courses for CT attenuation and change in $\mathrm{ADC}$ value. Our data reproduced the clinical result of different time dependencies between $\mathrm{CT}$ attenuation and ADC value. However, our data suggest that the severity of diffusion restriction is not linearly correlated with the net water uptake of acute ischemic tissue, which is manifested as a decrease in CT attenuation in our experimental animal model of acute cerebral ischemia. This finding may indicate that the pathophysiology of CT hypoattenuation in acute cerebral ischemia is different from that of ADC decrease. Although the exact reason for the discrepancy in results between our study and the previous clinical study by Kucinski et $\mathrm{al}^{27}$ is unclear, it may be related to differences in the relatively homogeneous cases of cerebral ischemia induced in experimental animal models and the heterogeneous severity and duration of ischemia injury of clinical ischemic stroke cases. In addition, the effects of anes- 


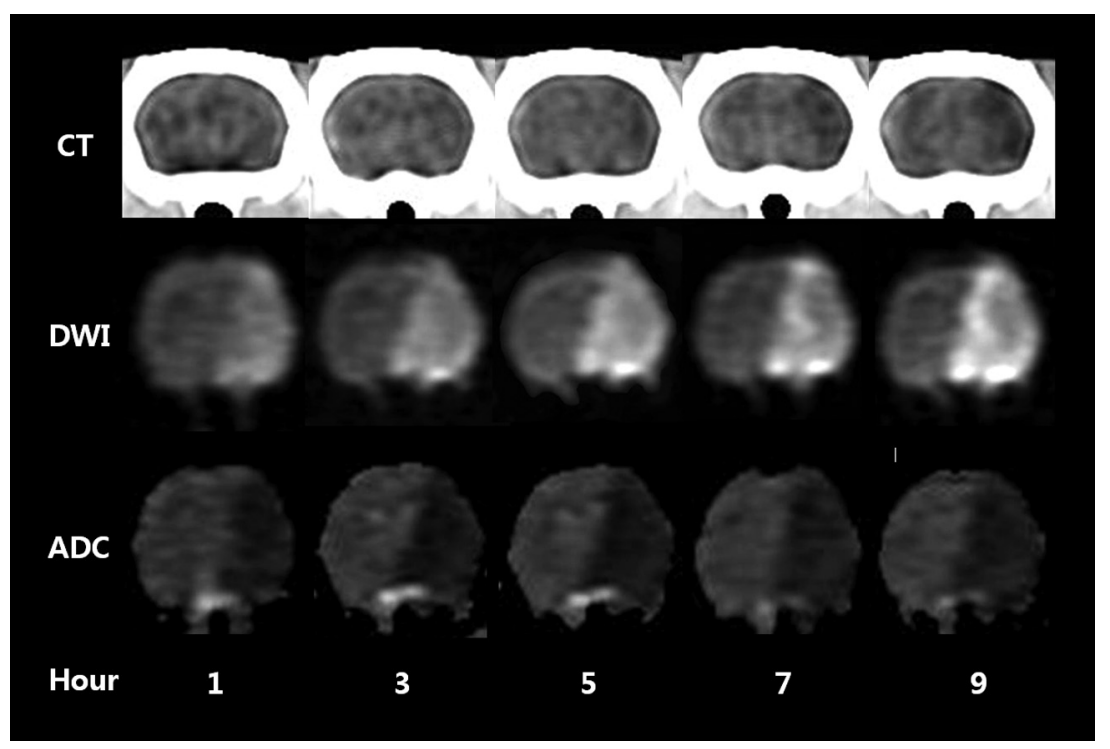

Fig 2. Longitudinal changes in acute ischemic lesions on $C T$, DWI, and the ADC map at each time point after MCA occlusion in a rat.

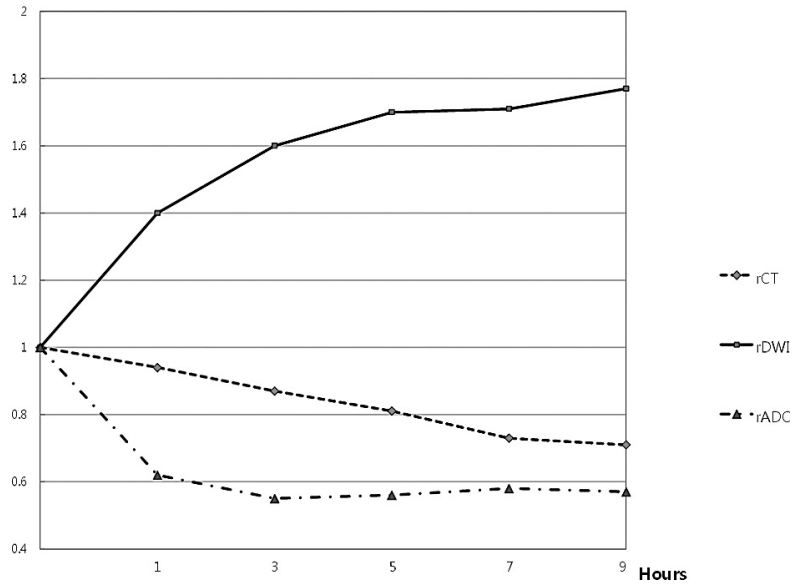

Fig 3. Time courses of rCT, rDWl, and rADC after MCA occlusion. The rCT decreases gradually and rDWI increases gradually with time $(P<.01$, respectively). However, rADC rapidly decreases at 1 hour after MCA occlusion, and rADC shows no substantial change after 3 hours $(P=.33)$

thesia, the relatively large ratio of gray to white matter in rat brain, and the difference of cerebral hemodynamics, including possible spontaneous reperfusion and collateral flow, may be other related factors.

CT attenuation on unenhanced CT correlates linearly with tissue-specific gravity ${ }^{29}$ and consequently correlates negatively with the net water amount of tissues. ${ }^{6,30}$ An increase of $1 \%$ water decreased CT attenuation by $2.6 \mathrm{HU}$ in 1 in vitro study with gel, ${ }^{31}$ and a decrease in CT attenuation of $1.8 \mathrm{HU}$ per $1 \%$ increase in tissue water was seen in an animal study. ${ }^{6}$ In acute cerebral infarction, decreased CT attenuation results from increased brain tissue water content, which occurs immediately after arterial occlusion ${ }^{3,4,32,33}$ and is continuous, ${ }^{6}$ consistent with the results of this study. The increase in net tissue water in acute infarction is thought of as a chain of water shift after cytotoxic edema. The water shift into the intracellular space in cytotoxic edema may generate an osmotic gradient between the extracellular and intravascular spaces, causing a gradual water shift into the extracellular space from the intra-

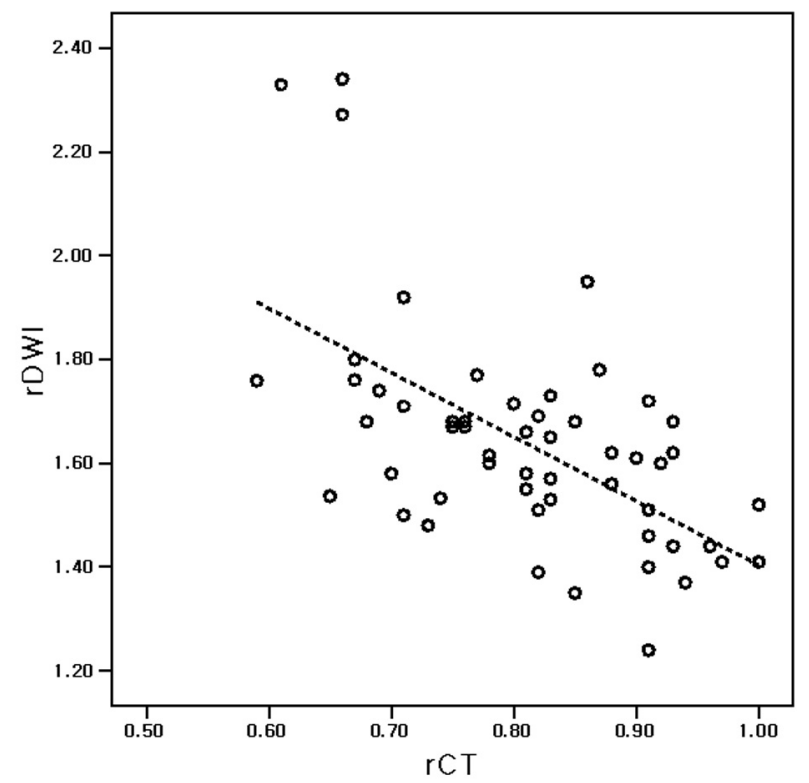

Fig 4. Scatterplot of rCT and rDWl. Significant correlation occurs between rCT and rDWl in acute cerebral ischemia after $1,3,5,7$, or 9 hours of MCA occlusion. The rCT and rDWl are inversely correlated $(P<.01, r=-0.592)$. The dotted line is the regression line for $\mathrm{rCT}$ and rDWI. One unit of rCT increase corresponds to $1.24 \mathrm{U}$ of rDWI decrease.

vascular space. ${ }^{34}$ Therefore, the water content of brain tissue increases and CT attenuation decreases in acute infarction before the blood-brain barrier is disrupted. ${ }^{3-5,32,33}$

Although the decrease of ischemic tissue in ADC is related to cytotoxic edema and energy failure ${ }^{35-39}$ and ADC changes in acute ischemic tissue are believed to be related to alterations in water compartments and more restricted water diffusion in the intracellular than the extracellular space, ${ }^{37-41}$ the exact mechanism of ADC decrease remains unclear. Other factors of temperature decrease and cell membrane permeability may play a role in the decrease of ADC in ischemic brain tissue. ${ }^{42-44}$

Although ADC decrease in ischemic tissue may be related to net water uptake during the early period of $<3$ hours of cerebral ischemia, ${ }^{45}$ our data do not support a linear relation- 


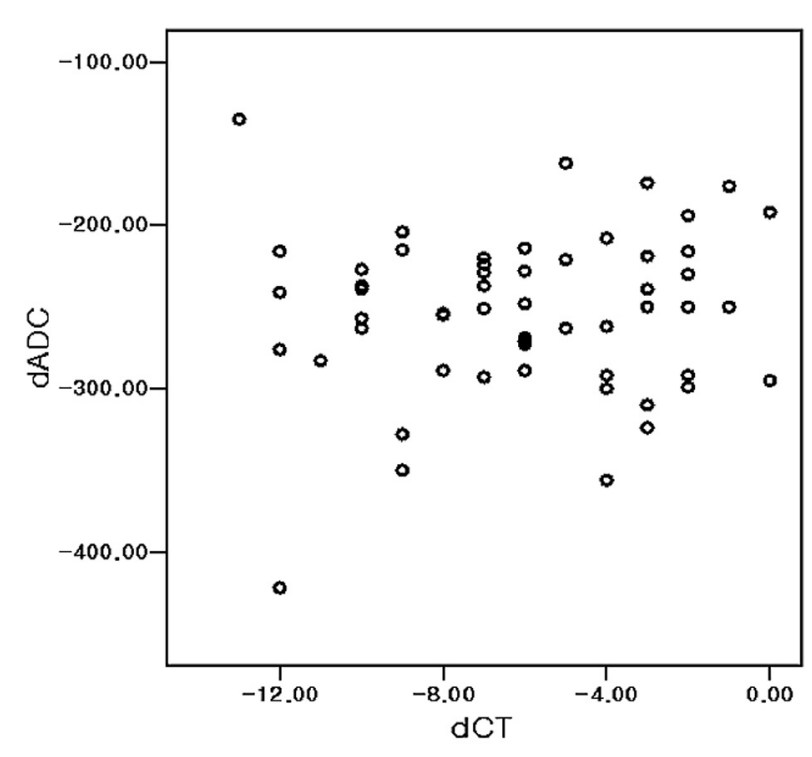

Fig 5. Scatterplot of $d C T$ and $d A D C$. No significant linear correlation occurs between $d A D C$ and $\mathrm{dCT}(P=.56)$ in acute cerebral ischemia after $1,3,5,7$, or 9 hours of MCA occlusion.

ship between ADC decrease and net water uptake in acute ischemic tissue. Kucinski et $\mathrm{al}^{46}$ demonstrated a correlation between relative $\mathrm{CBF}$ and decrease in $\mathrm{CT}$ or $\mathrm{ADC}$ in acute ischemic stroke, and the reduction in regional CBF was suggested as a common underlying reason for the phenomena of decreased CT attenuation and ADC in acute ischemic tissue. ${ }^{27}$ We agree that the regional CBF reduction is a common cause of ischemic tissue injury and that both CT attenuation and ADC reflect brain tissue changes after acute ischemic injury. We believe that CT and ADC represent different aspects and phenomena of acute ischemic tissue induced by CBF reduction. The linear correlation between CT attenuation and brain tissue water uptake suggests that the severity of acute ischemic edema can be monitored with CT. ${ }^{6}$ The nonlinear time course of ADC decrease and the lack of linear correlation between $\mathrm{ADC}$ and $\mathrm{CT}$ attenuation in ischemic tissue indicated that the severity of ADC reduction did not have a close linear relationship to the severity of acute ischemic edema. A previous study by Kim et $\mathrm{al}^{19}$ reported that clinical acute ischemic stroke cases show reverse discrepancy between CT and DWI, which might be a phenomenon of transient early reversal of ADC reduction by early reperfusion. This study suggested that the severe ischemic edema seen as CT hypoattenuation might not result in a reduction in $\mathrm{ADC}$.

The DWI signal intensity depends on the ADC value and T2 signal intensity, and tissue water in brain edema strongly correlates with T2 signal intensity, which is not necessarily sensitive for detecting the cytotoxic edema without disruption of blood-brain barrier and macromolecules originating from the blood serum. ${ }^{4-50}$ The increase of tissue water may explain the significant correlation between CT attenuation and DWI signal intensity in acute ischemic tissue.

The mechanism of the longitudinal change pattern of ADC decrease after ischemic stroke, early rapid ADC decrease and plateau, is not clear. However, our data of the phenomenon of $\mathrm{ADC}$ and DWI signal intensity change in acute ischemic tissue may provide some information in understanding of DWI in acute ischemic stroke. First, ADC decrease does not have a close linear relationship with the severity of brain tissue injury (brain edema manifest as hypoattenuation on CT), because ischemic damage of brain tissue progresses after ischemic stroke in persistent occlusion modes. This finding may support the results of previous studies ${ }^{51,52}$ that there seems no close correlation between neuronal damage and $\mathrm{ADC}$ value. Second, therefore, ADC value seems not highly specific for tissue viability, which also supports the results of clinical studies $^{18,53-55}$ that ADC is not a reliable predictor of irreversible tissue damage. Third, DWI signal intensity may be more predictive of the severity of ischemic injury because DWI signal intensity continuously increases after ischemic stroke and has a correlation with CT attenuation and ischemic edema. This also supports the opinion derived from previous animal studies that DWI signal intensity may be a better marker of neuronal death than ADC. ${ }^{52}$ Our data suggest that the severity of $\mathrm{ADC}$ decrease alone may not reliably predict the severity of ischemic edema in the time window for thrombolytic therapy, especially beyond the early period after 3 hours of ischemic injury. Our study suggests that cautious interpretation of DWI is needed in the estimation of the severity of ischemic injury and predicting the risk of thrombolytic therapy in acute ischemic stroke.

The early decrease in ADC allows an early detection of acute infarction, while the gradual and minimal decrease in CT attenuation lowers the sensitivity of CT for hyperacute ischemic edema compared with DWI. Our data showing a lower CNR for CT attenuation also explains the relatively lower sensitivity of CT for detecting early ischemic change. The higher CNR for DWI signal intensity suggests that DWI is more sensitive in the detection of acute cerebral infarction than CT or an ADC map.

This study had several limitations. First, it included a small number of animals. Second, we could not perform pathologic correlation of the severity of ischemic tissue injury with imaging findings. Third, CT and DWI were not spatially coregistered for region-of-interest analysis. However, we used relatively large regions of interest and carefully selected images by using anatomic landmarks to minimize the possible errors by noncoregistration.

\section{Conclusions}

This longitudinal imaging study of acute cerebral infarction in a rat model shows different time courses for $\mathrm{CT}$ attenuation and $\mathrm{ADC}$ value. However, $\mathrm{rCT}$ and $\mathrm{rDWI}$ showed a modest correlation. Although DWI or ADC were more sensitive for detecting acute ischemic lesions, DWI may be a more reliable predictor of the severity of ischemic edema than the ADC value beyond 3 hours in this experimental animal stroke model.

\section{References}

1. von Kummer R, Bourquain H, Bastianello S, et al. Early prediction of irreversible brain damage after ischemic stroke at CT. Radiology 2001;219:95-100

2. Grond M, von Kummer R, Sobesky J, et al. Early $\mathbf{x}$-ray hypoattenuation of brain parenchyma indicates extended critical hypoperfusion in acute stroke. Stroke 2000;31:133-39

3. Schuier FJ, Hossmann KA. Experimental brain infarcts in cats. II. Ischemic brain edema. Stroke 1980;11:593-601

4. Todd N, Picozzi P, Crockard A, et al. Duration of ischemia influences the development and resolution of ischemic brain edema. Stroke 1986;17:466-71 5. Gotoh O, Asano T, Koide T, et al. Ischemic brain edema following occlusion of 
the middle cerebral artery in the rat. I. The time courses of the brain water, sodium, and potassium contents and blood-brain-barrier permeability to 125I-albumin. Stroke 1985;16:101-09

6. Dzialowski I, Weber J, Doerfler A, et al. Brain tissue water uptake after middle cerebral artery occlusion assessed with CT. J Neuroimaging 2004;14:42-48

7. von Kummer R, Allen K, Holle R, et al. Acute stroke: usefulness of early CT findings before thrombolytic therapy. Radiology 1997;205:327-33

8. Barber P, Demchuk A, Zhang J, et al. Validity and reliability of a quantitative computed tomography score in predicting outcome of hyperacute stroke before thrombolytic therapy. Lancet 2000;355:1670-74

9. Tanne D, Kasner S, Demchuk A, et al. Markers of increased risk of intracerebral hemorrhage after intravenous recombinant tissue plasminogen activator therapy for acute ischemic stroke in clinical practice. Circulation 2002;105:1679-85

10. Gonzalez RG, Schaefer PW, Buonanno FS, et al. Diffusion-weighted MR imaging: diagnostic accuracy in patients imaged within 6 hours of stroke symptom onset. Radiology 1999;210:155-62

11. Mohr JP, Biller J, Hilal SK, et al. Magnetic resonance versus computed tomographic imaging in acute stroke. Stroke 1995;26:807-12

12. Kohno K, Hoehn-Berlage M, Mies G, et al. Relationship between diffusion weighted MR images, cerebral blood flow, and energy state in experimental brain infarction. Magn Reson Imaging 1995;13:73-80

13. Lin W, Lee JM, Lee YZ, et al. Temporal relationship between apparent diffusion coefficient and absolute measurements of cerebral blood flow in acute stroke patients. Stroke 2003;34:64-67

14. Kidwell CS, Saver JL, Mattiello J, et al. Thrombolytic reversal of acute human cerebral ischemic injury shown by diffusion/perfusion magnetic resonance imaging. Ann Neurol 2000;47:462-69

15. Neumann-Haefelin T, Kastrup A, de Crespigny A, et al. Serial MRI after transient focal cerebral ischemia in rats: dynamics of tissue injury, blood-brain barrier damage, and edema formation. Stroke 2000;31:1965-72

16. Li F, Liu KF, Silva MD, et al. Acute postischemic renormalization of the apparent diffusion coefficient of water is not associated with reversal of astrocytic swelling and neuronal shrinkage in rats. AJNR Am J Neuroradiol 2002; $23: 180-88$

17. Olah L, Wecker S, Hoehn M. Relation of apparent diffusion coefficient changes and metabolic disturbances after 1 hour of focal cerebral ischemia and at different reperfusion phases in rats. J Cereb Blood Flow Metab 2001;21:430-39

18. Fiehler J, Foth M, Kucinski T, et al. Severe ADC decreases do not predict irreversible tissue damage in humans. Stroke 2002;33:79-86

19. Kim EY, Ryoo JW, Roh HG, et al. Reversed discrepancy between CT and diffusion-weighted MR imaging in acute ischemic stroke. AJNR Am J Neuroradiol. 2006;27:1990-95

20. Davis D, Ulatowski J, Eleff S, et al. Rapid monitoring of changes in water diffusion coefficients during reversible ischemia in cat and rat brain. Magn Reson Med 1994;31:454-60

21. Hoehen-Berlage M, Eis M, Back T, et al. Change of relaxation times (T1, T2) and apparent diffusion coefficient after permanent middle cerebral artery occlusion in the rat: temporal evolution, regional extent, and comparison with histology. Magn Reson Med 1995;34:824-34

22. Pierapaoli C, Righini A, Linfante I, et al. Histopathologic correlates of abnormal water diffusion in cerebral ischemia: diffusion-weighted MR imaging and light and electron microscopic study. Radiology 1993;189:439-48

23. Gerriets T, Stolz E, Walberer M, et al. Middle cerebral artery occlusion during MR-imaging: investigation of the hyperacute phase of stroke using a new inbore occlusion model in rats. Brain Res 2004;12:137-43

24. Kastrup A, Engelhorn T, Beaulieu C, et al. Dynamics of cerebral injury, perfusion, and blood-brain barrier changes after temporary and permanent middle cerebral artery occlusion in the rat. J Neurol Sci 1999;166:91-99

25. Lutsep H, Albers G, de Crespigny A, et al. Clinical utility of diffusion-weighted magnetic resonance imaging in the assessment of ischemic stroke. Ann Neurol 1997;41:574-80

26. Schlaug G, Siewert B, Benfield BS, et al. Time course of the apparent diffusion coefficient (ADC) abnormality in human stroke. Neurology 1997;49:113-19

27. Kucinski T, Vaterlein O, Glauche V, et al. Correlation of apparent diffusion coefficient and computed tomography density in acute ischemic stroke. Stroke 2002;33:1786-91

28. Longa EZ, Weinstein PR, Carlson S, et al. Reversible middle cerebral artery occlusion without craniectomy in rats. Stroke 1989;20:84-91

29. Torack RM, Alcala H, Gado M, et al. Correlative assay of computerized cranial tomography CCT, water content and specific gravity in normal and pathological postmortem brain. J Neuropathol Exp Neurol 1976;35:385-92
30. Rieth KG, Fujiwara K, Di Chiro G, et al. Serial measurements of CT attenuation and specific gravity in experimental cerebral edema. Radiology 1980;135:343-48

31. Unger E, Littlefield J, Gado M. Water content and water structure in CT and MR signal changes: possible influence in detection of early stroke. AJNR Am J Neuroradiol 1988;9:687-91

32. Watanabe O, West CR, Bremer A. Experimental regional cerebral ischemia in the middle cerebral artery territory in primates. Part 2. Effects on brain water and electrolytes in the early phase of MCA stroke. Stroke 1977;8:71-76

33. Menzies SA, Betz AL, Hoff JT. Contributions of ions and albumin to the formation and resolution of ischemic brain edema. J Neurosurg 1993;78:257-66

34. Go KG. The normal and pathological physiology of brain water. Adv Tech Stand Neurosurg 1997;23:47-142

35. Sevick RJ, Kanda F, Mintorovitch J, et al. Cytotoxic brain edema: assessment with diffusion-weighted MR imaging. Radiology 1992;185:687-90

36. Moseley ME, Cohen Y, Mintorovitch J, et al. Early detection of regional cerebral ischemia in cats: comparison of diffusion- and T2-weighted MRI and spectroscopy. Magn Reson Med 1990;14:330-46

37. Wick M, Nagatomo Y, Prielmeier F, et al. Alteration of intracellular metabolite diffusion in rat brain in vivo during ischemia and reperfusion. Stroke 1995;26:1930-33

38. van der Toorn A, Dijkhuizen RM, Tulleken CA, et al. Diffusion of metabolites in normal and ischemic rat brain measured by localized 1H MRS. Magn Reson Med 1996;36:914-22

39. Duong TQ, Ackerman JJ, Ying HS, et al. Evaluation of extra- and intracellular apparent diffusion in normal and globally ischemic rat brain via 19F NMR. Magn Reson Med 1998;40:1-13

40. Mintorovitch J, Yang GY, Shimuzu H, et al. Diffusion-weighted magnetic resonance imaging of acute focal cerebral ischemia: comparison of signal intensity with changes in brain water and $\mathrm{Na}+, \mathrm{K}(+)$-ATPase activity. JCereb Blood Flow Metab 1994;14:332-36

41. Benveniste H, Hedlund LW, Johnson GA. Mechanism of detection of acute cerebral ischemia in rats by diffusion-weighted magnetic resonance microscopy. Stroke 1992;23:746-54

42. Morikawa E, Gingsberg MD, Dietrich WD, et al. The significance of brain temperature in focal cerebral ischemia: histopathological consequences of middle cerebral artery occlusion in the rat. J Cereb Blood Flow Metab 1992;12:380-89

43. Le Bihan D, Delannoy J, Levin RL. Temperature mapping with MR imaging of molecular diffusion: application to hyperthermia. Radiology 1989;171:853-57

44. Szafer A, Zhong J, Gore JC. Theoretical model for water diffusion in tissues. Magn Reson Med 1995;33:697-712

45. Kuroiwa T, Nagaoka T, Ueki M, et al. Different apparent diffusion coefficient: water content correlations of gray and white matter during early ischemia. Stroke 1998;29:859-65

46. Kucinski T, Majumder A, Knab R, et al. Cerebral perfusion impairment correlates with the decrease of CT density in acute ischaemic stroke. Neuroradiology 2004;46:716-22

47. Kato H, Kogure K, Ohtomo H, et al. Characterization of experimental ischemic brain edema utilizing proton nuclear magnetic resonance imaging. J Cereb Blood Flow Metab 1986;6:212-21

48. Boisvert DP, Handa Y, Allen PS. Proton relaxation in acute and subacute ischemic brain edema. Adv Neurol 1990;52:407-13

49. Qiao M, Malisza KL, Del Bigio MR, et al. Correlation of cerebral hypoxicischemic T2 changes with tissue alterations in water content and protein extravasation. Stroke 2001;32:958-63

50. Naruse S, Horikawa Y, Tanaka C, et al. Proton nuclear magnetic resonance studies on brain edema. J Neurosurg 1982;56:747-52

51. Cvoro V, Marshall I, Armitage PA, et al. MR diffusion and perfusion parameters: relationship to metabolites in acute ischaemic stroke. J Neurol Neurosurg Psychiatry 2010;81:185-91

52. Rivers CS, Wardlaw JM. What has diffusion imaging in animals told us about diffusion imaging in patients with ischaemic stroke? Cerebrovasc Dis 2005; 19:328-36

53. Loh PS, Butcher KS, Parsons MW, et al. Apparent diffusion coefficient thresholds do not predict the response to acute stroke thrombolysis. Stroke 2005;36:2626-31

54. Nicoli F, Lefur Y, Denis B, et al. Metabolic counterpart of decreased apparent diffusion coefficient during hyperacute ischemic stroke: a brain proton magnetic resonance spectroscopic imaging study. Stroke 2003;34:e82-87

55. Guadagno JV, Warburton EA, Aigbirhio FI, et al. Does the acute diffusionweighted imaging lesion represent penumbra as well as core? A combined quantitative PET/MRI voxel-based study. J Cereb Blood Flow Metab 2004;24:1249-54 